\title{
Proposal of peritoneal biopsy procedures for patients undergoing peritoneal dialysis
}

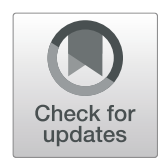

\author{
Tohru Mizumasa ${ }^{1 *}$, Kazuho Honda $^{2}$, Shigehisa Aoki ${ }^{3}$, Chieko Hamada $^{4}$, Masanobu Miyazaki ${ }^{5}$, Yasuhiko Ito ${ }^{6}$, \\ Yudo Tanno ${ }^{7}$, Toshiaki Nakano ${ }^{8}$, Masaaki Nakayama ${ }^{9}$ and on behalf of the Peritoneal Biopsy Study Group of the \\ Japanese Society for Peritoneal Dialysis
}

\begin{abstract}
Prolonged peritoneal dialysis (PD) is responsible for progressive morphological changes such as deterioration of the peritoneal membrane. These changes increase the risk of encapsulating peritoneal sclerosis (EPS). Histological assessments of peritoneal membrane biopsy samples are fundamental for the evaluation of the peritoneal damage caused by PD. For evaluating serial morphological changes induced in the peritoneum by PD, we recommend to perform peritoneal biopsy examinations not only after the cessation of PD but also before performing PD. At the time of PD catheter insertion, the parietal peritoneum $(1.5 \times 1.5 \mathrm{~cm})$ and rectus abdominal muscle posterior sheath is sampled at a point $3 \mathrm{~cm}$ below the PD catheter insertion site. Furthermore, at the time of PD catheter removal, the parietal peritoneum is sampled at a point $3 \mathrm{~cm}$ apart from the PD catheter insertion site to avoid artifacts. The peritoneum should be evaluated to detect mesothelial cell denudation, acellular sclerotic changes and thickness of the submesothelial connective tissue, vasculopathy of the post-capillary venules, vascular angiogenesis, and new encapsulating membrane. The method presented herein allows the minimization of surgical invasiveness and artifacts of the specimens and safe performance of peritoneal biopsy examinations. Morphological evaluation of the peritoneum involving an appropriate biopsy strategy, in conjunction with functional markers of deterioration, such as peritoneal permeability or cytokine levels, is a useful approach for examining peritoneal damage and predicting the prognosis of PD patients, especially the onset of EPS.
\end{abstract}

Keywords: Peritoneal biopsy, Artifact, Vasculopathy, Angiogenesis, Encapsulating membrane, EPS

\section{Background}

With the prolongation of peritoneal dialysis (PD) therapy, morphological changes such as deterioration of the peritoneal membrane occur [1-3], increasing the risk for encapsulating peritoneal sclerosis (EPS) [4-6]. At our hospital, peritoneal biopsy examinations are performed not only after the cessation of PD but also before the initiation of PD to evaluate the serial morphological changes induced by PD in the peritoneum. A unified method for performing peritoneal biopsy remains to be established because, to date, few studies have reported on its methodology [7-9]. Therefore, the technique for a peritoneal biopsy varies across institutions. Reportedly, an accurate evaluation is complicated by the absence of

\footnotetext{
* Correspondence: jac-man7@ja2.so-net.ne.jp

${ }^{1}$ Department of Nephrology, Kyushu Central Hospital of the Mutual Aid

Association of Public School Teachers, Fukuoka, Japan

Full list of author information is available at the end of the article
}

an appropriate sampling technique, especially in cases with a peritoneum of almost normal thickness, which is prone to mechanical damages [7]. The thickness of the peritoneum prior to performing PD is around $100 \mu \mathrm{m}$; however, the degree of thickness becomes remarkable with the prolongation of PD. Recently, new neutral-pH, low-glucose degradation product (GDP) PD solutions have been developed to substitute conventional acidic PD fluids, and reports have shown that peritoneal thickening induced by the neutral PD solutions is milder than that induced by acidic PD solutions [3]. Hence, special attention to peritoneal biopsy is required not only before performing PD but also after the cessation of PD therapy. Moreover, morphological evaluation of the peritoneum may become difficult due to factors such as wound healing around PD catheter insertion site, differences in the collection area, and supportive connective tissue including fascia. Therefore, in the present paper that is the

(c) The Author(s). 2020 Open Access This article is distributed under the terms of the Creative Commons Attribution 4.0 International License (http://creativecommons.org/licenses/by/4.0/), which permits unrestricted use, distribution, and 
position paper from the Japanese Society for Peritoneal Dialysis, we propose a safe method of performing peritoneal biopsy that minimizes surgical invasiveness and sample artifacts. Additionally, we have commented on points to be noted.

\section{Significance of peritoneal biopsy}

Prolonged PD leads to morphological changes in the peritoneal membrane; these include denudation of mesothelial cells, thickening, and sclerotic changes in submesothelial connective tissue, vasculopathy, angiogenesis, and new membrane formation on the existing peritoneum [1-3]. These morphological changes may lead to high permeability of not only small (such as glucose, urea, creatinine, and electrolytes) but also large (such as fibrinogen and various coagulation factors) molecules, resulting in an increased risk for EPS. Histological assessments of the peritoneal membrane in biopsy samples are fundamental for the evaluation of the peritoneal damage caused by $\mathrm{PD}$, as well as for the diagnosis and prediction of EPS.

\section{Past reports and issues about peritoneal biopsy}

To the best of our knowledge, only a few studies have reported on the methodology of peritoneal biopsy [710]. Moreover, a detailed biopsy procedure has been described in only one study [7], where the authors have proposed a suture method for collecting peritoneal samples. Briefly, a suture is inserted into the outer part of the abdominal musculature, and the area is raised to avoid any accidental damage to the underlying organs. A portion of the abdominal wall musculature and the attached peritoneum is excised together with the suture. The specimen is briefly placed in a sample buffer before being pinned onto a corkboard, with the mesothelial surface being on the top. However, we believe that this method may cause some problems. First, this method holds the potential risk for injuring internal organs, especially at the time of suture and excision, because it is impossible to visually detect a possible adhesion between the abdominal wall and internal organs. Second, pinning to a corkboard may cause peritoneal specimens to get fixed in an excessively pulled form. Moreover, this procedure may give rise to issues such as a complex preparation and a prolonged time required for biopsy. Third, morphological evaluations of the marginal portion of the specimen may contain artifacts following the damage caused by pinning or by touching with micro tweezers to perform the pinning. Moreover, the evaluation of the central part of the specimen may become difficult if the suture is unexpectedly deep.

\section{Proposal of peritoneal biopsy procedure Operation instrument}

We use Mosquito-pean forceps to exfoliate the rectus abdominis muscle posterior sheath and peritoneum (Fig. 1a; Kaminaka-type forceps: Kyusyu Fuundo Co., Japan. Type number: J314/SHT); the tip of these forceps is very thin (about $1 \mathrm{~mm}$ ). Similarly, we use Mosquito Kocher forceps to grip the peritoneum (Fig. 1b; Monsieur forceps: Taiyu Medical Industry Co., Japan. Type number:
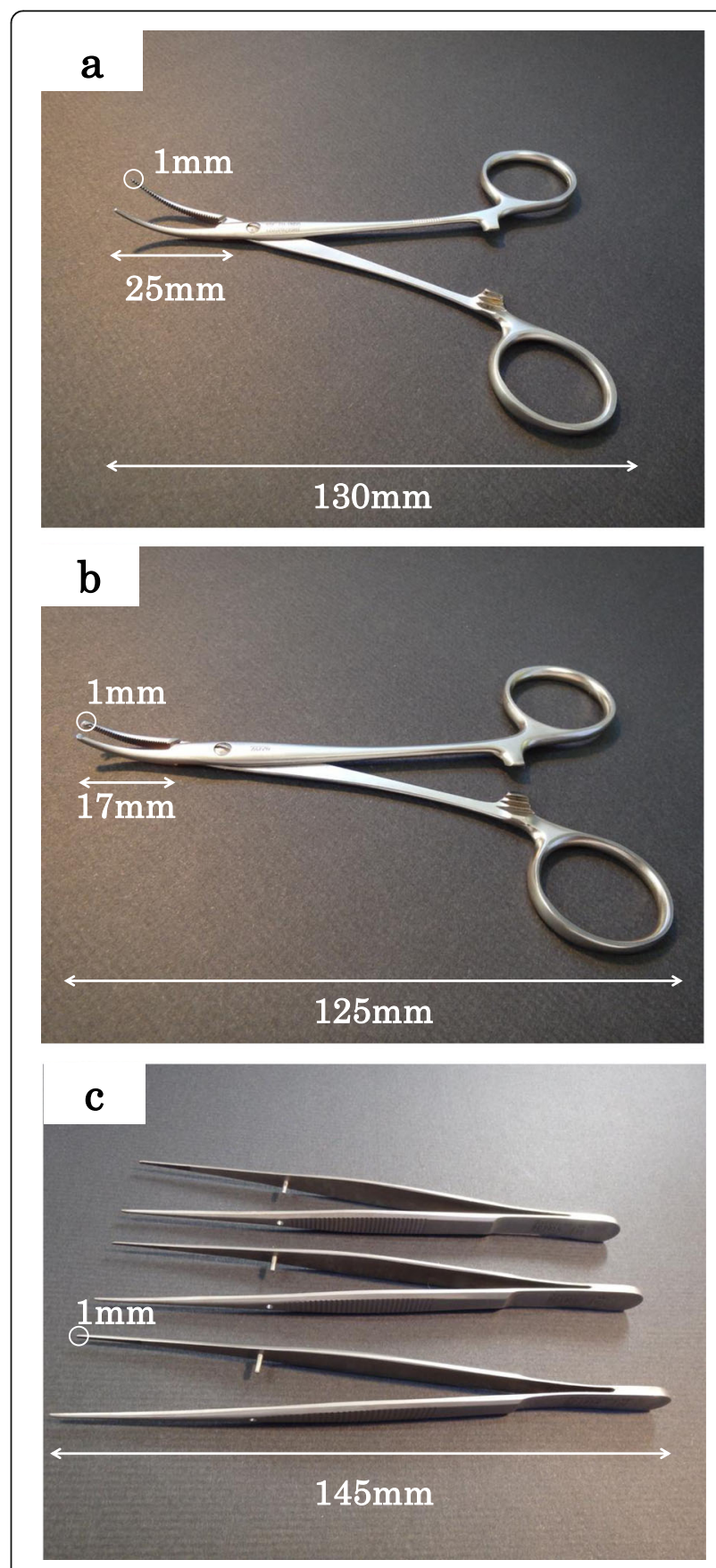

Fig. 1 Tools used for peritoneal biopsy.a Mosquito-pean forceps. b Mosquito Kocher forceps. c Micro tweezers 
TY-428B) and micro tweezers (Fig. 1c; B Braun Aesculap Co., Japan. Type number: BD305R), the thin tip of which is preferable to treat the specimen. Because these instruments are only representative examples, we believe that there will not be any problem if similar instruments are used. Furthermore, clean cellophane to place the specimen and a vessel containing 10\% formaldehyde as fixative should be prepared.

\section{Surgical procedure for peritoneal biopsy at PD catheter insertion}

The parietal peritoneum is sampled at the time of PD catheter insertion. After making a paramedian incision at a point twice, the width of a finger lateral to the umbilicus, the rectus abdominis muscle anterior sheath is incised. Following this, the rectus abdominis muscle is separated to expose the posterior sheath and peritoneum. To avoid damages of the peritoneum due to air drying, we recommend that biopsy is performed prior to the subsequent surgical procedure. The parietal peritoneum with the rectus abdominis muscle posterior sheath is collected at a point $3 \mathrm{~cm}$ below the PD catheter insertion site. The rectus abdominis muscle posterior sheath and peritoneum are gripped together using Mosquito Kocher forceps and then exfoliated using Mosquito-pean forceps to reach the abdominal cavity (Fig. 2a). Notably, Mosquito-pean forceps should be carefully operated at the same pinpoint, without greatly moving them for exfoliation. In cases where the exfoliation of the peritoneum is difficult, a small incision may be made on the peritoneum using a scalpel while taking care to avoid any damage to the internal organs due to adhesion with the abdominal wall. The edge of the biopsy area is held at the shallow edge using the Mosquito Kocher forceps. This point is considered the starting point for the biopsy (Fig. 2b). An incision is made at starting points at both
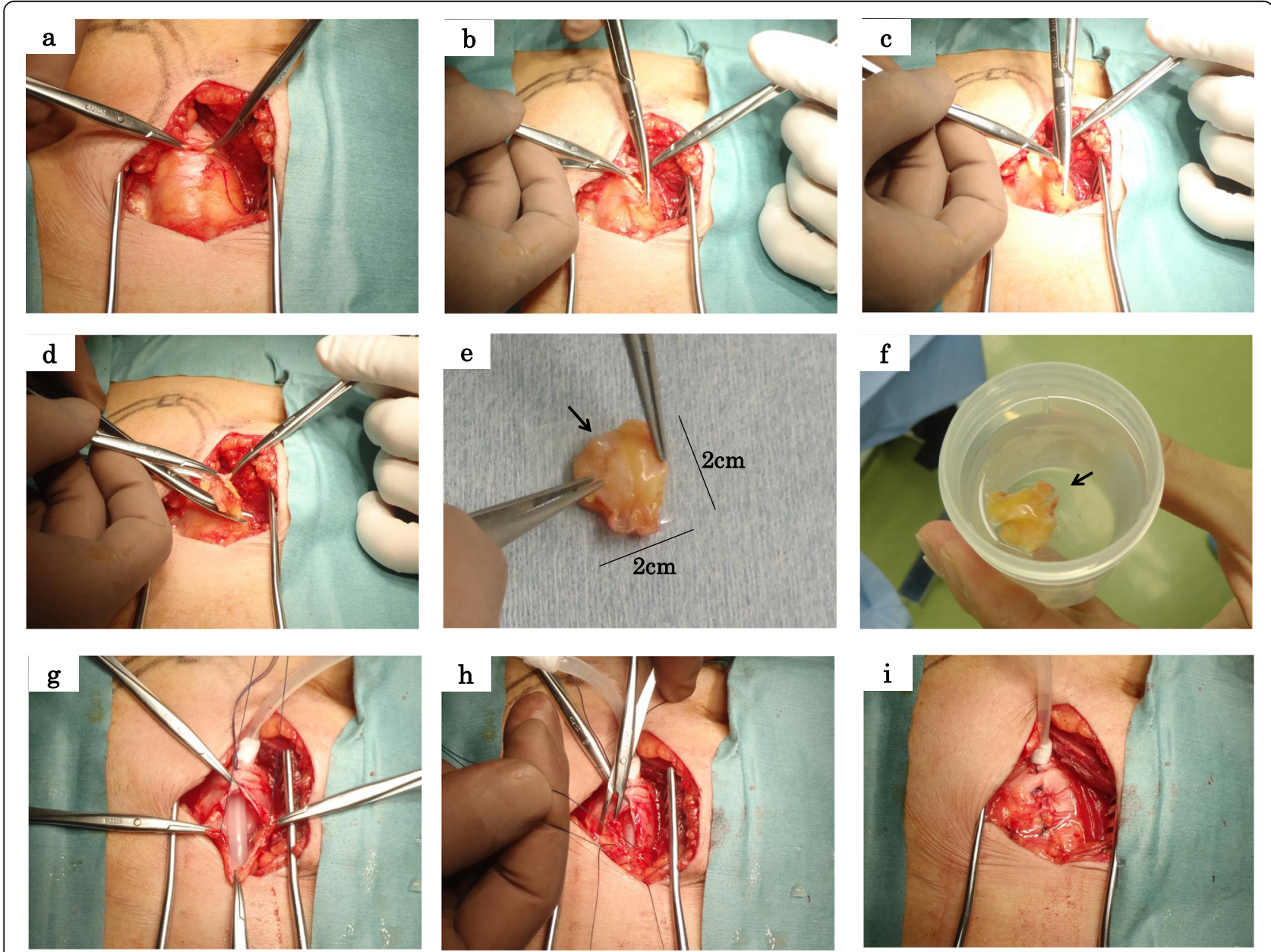

Fig. 2 Surgical procedure for peritoneal biopsy. These figures were quoted and modified from reference [9]. a A picture when reaching the abdominal cavity. b, c Pictures when making an incision at the starting points and while progressing in a slightly outward direction. $\mathbf{d}$ A picture when the specimen was separated from the body. e, $\mathbf{f}$ The parietal peritoneum sampled. Arrows show sampled peritoneum developed on a $2 \times$ $2 \mathrm{~cm}$ section of cellophane. $\mathbf{g}$-i Peritoneal wall anchor technique by using the biopsy area 
ends of the Mosquito Kocher forceps while taking care not to touch the peritoneal surface. Of note, this incision is made in a slightly outward direction to obtain an adequate amount of specimen (Fig. 2c). The specimen is separated from the body by gripping it with Mosquito Kocher forceps (Fig. 2d). Subsequently, the parietal peritoneum $(1.5 \times 1.5 \mathrm{~cm})$ and rectus abdominis muscle posterior sheath are collected. The specimen is placed on a $2 \times 2 \mathrm{~cm}$ section of cellophane such that the posterior sheath faces the cellophane's surface and the mesothelial surface is on the top. The part of the specimen that has been gripped or touched is not suitable for morphological evaluation; thus, an adequate amount of specimen is obtained. Additionally, the part of the specimen $>1 \mathrm{~mm}$ from the edge toward the center should not be touched when placing on the cellophane using micro tweezers (Fig. 2e). Because prolonged exposure of the peritoneum to air leads to mesothelial cell degeneration, we minimize the duration between sampling and placing the tissue into the fixative to within $5 \mathrm{~min}$. The specimen is placed into the fixative with the cellophane side above, taking care to ensure that the cellophane does not come off (Fig. 2f). Finally, the biopsy area is closed finely using absorbable sutures to avoid leakage of the dialysate. At our institution, the PD catheter is inserted at a point $3 \mathrm{~cm}$ cranial to the biopsy area and is then fixed to the abdominal wall using a 2-0 nylon suture under vision from the biopsy area (peritoneal wall anchor technique) (Fig. 2g-i) [11]. The peritoneal wall anchor technique reportedly improves the catheter survival rate [10], although the catheter is blindly fixed to the abdominal wall at the area inferior to the catheter insertion in the absence of peritoneal biopsy. However, in addition to the aforementioned advantage [11], the present method employing biopsy area allows for the fixation of the catheter to the abdominal wall in a safe and simple manner.

\section{Surgical procedure for peritoneal biopsy at PD catheter removal}

The biopsy procedure for peritoneum at PD catheter removal is similar to that at PD catheter insertion. A paramedian incision is made at the same location as that of the operation scar for catheter insertion. For ease of performing the biopsy, we recommend extending the skin incision to approximately $1 \mathrm{~cm}$ lower than the operation scar. Following the exfoliation of the subcutaneous adipose tissue, the rectus abdominis muscle anterior sheath is incised. Subsequently, the rectus abdominis muscle is separated to expose the posterior sheath and peritoneum. Because the PD catheter insertion area is believed to be affected by the operation and wound healing at the PD catheter insertion, peritoneal biopsy is performed more than $3 \mathrm{~cm}$ apart from the insertion site. That is, a large incision is made in the anterior sheath of the rectus abdominis muscle from the areas inferior to the area in which the deep cuff is placed such that the rectus abdominis muscle is bluntly dissected to reach the rectus abdominis muscle posterior sheath. Alternatively, the anterior sheath is incised at another area lateral and inferior to the area in which the deep cuff is detained. To avoid damages of the peritoneum due to air drying, a biopsy is performed prior to the PD catheter removal procedure. A subsequent biopsy procedure is performed as described above (Fig. 3). PD catheter removal is performed after the biopsy. To safely perform the removal, the abdominal cavity around the PD catheter insertion area is observed through the hole made for performing the biopsy to confirm the presence of adhesion between the intra-abdominal organs and PD catheter. Furthermore, the peritoneal cavity can be visualized through the biopsy area (i.e., adhesion or deterioration of the peritoneum) in a visible range.

\section{Points to be noted at biopsy}

We recommend that the following parameters at peritoneal biopsy are noted. (1) To prevent the distortion and shrinkage of the peritoneum, it is sampled with the rectus abdominis muscle posterior sheath. (2) The peritoneum is sampled prior to PD catheter insertion or removal to avoid prolonged exposure to air. (3) The peritoneum is sampled without direct contact and electrocautery to avoid mechanical injury. (4) The specimen is placed on cellophane without excessive stretching so

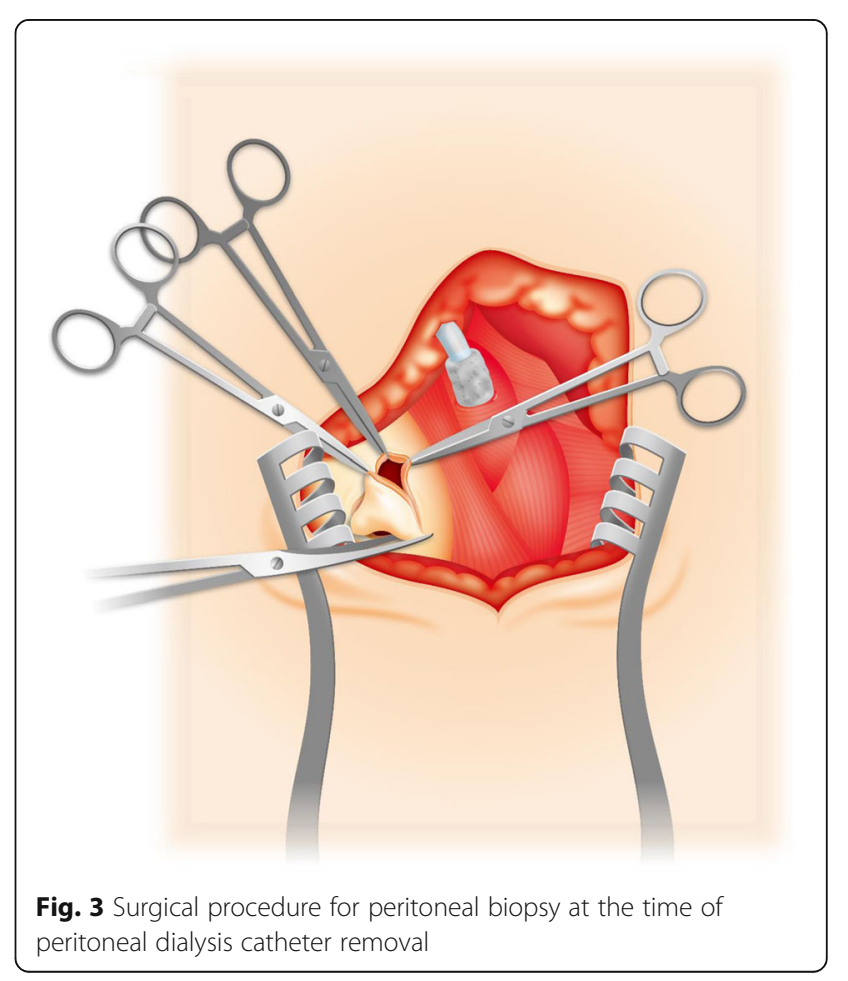


that the posterior sheath faces the cellophane's surface and mesothelial surface is on the top. (5) To avoid detachment from the cellophane, the operator places the specimen in the fixative carefully. (6) Peritoneal sampling procedure time is within 5 min.

In an earlier report, it has been shown that the specimen should be pinned onto a corkboard, with the mesothelial surface being on the top, after being briefly placed in sample buffer [7]. However, in our experience, the actual evaluation of the specimen is difficult because of its forcible fixation. Hence, as explained above, we currently use the cellophane; however, the specimen must be treated with care so as not to detach the specimen from the cellophane when placing the specimen into the fixation buffer.

\section{Histological analyses}

The sample is fixed at room temperature for $24 \mathrm{~h}$ in $10 \%$ phosphate-buffered formalin, embedded in paraffin, cut into $3-\mu \mathrm{m}$ sections, and fixed on a glass slide. Hematoxylin and eosin staining, Masson trichrome staining, and Elastica van Gieson staining are generally performed.

The purpose of a peritoneal biopsy is to evaluate the degree of peritoneal damage caused by PD and to evaluate the risk for EPS progression. The evaluation items include denudation of and morphological changes in mesothelial cells $[1,8]$, thickness and degeneration of submesothelial connective tissue [1-3, 8, 12-18], degree of vasculopathy $[1-3,8,12-20]$, angiogenesis $[1,8,12$, 14-17, 20-22], and presence of new membrane formation on the existing peritoneum $[15,17]$. The thickness of the submesothelial connective tissue is usually measured based on the thickness of the so-called submesothelial compact zone (SCZ) immediately below the mesothelial cell layer to the fatty tissue layer. The evaluated value includes the following: the mean of SCZ thickness at several randomly selected sites $[2,3,8,12-$ 15], maximum SCZ thickness [1], mean of the maximum and minimum SCZ thickening degrees [16, 17], or mean of whole SCZ thickness [18]. Post-capillary venule (PCV), which normal size is from 25 to $50 \mu \mathrm{m}$, is usually used to evaluate vasculopathy. The evaluated value of vasculopathy includes a semi-quantitative grading system (grade 0-3) based on the following: vascular wall thickening and vascular lumen stenosis [1, 8, 16-19], the ratio of luminal diameter to vessel external diameter (L/ $\mathrm{V}$ ratio) $[2,3,12-15,18]$, or the ratio of wall to total area [20]. Angiogenesis is usually evaluated as microvascular density using an optical microscope. The evaluated value includes the following: the number of blood vessels per unit area of SCZ $[12,14,16,17,20,21]$, number of blood vessels per unit length of SCZ $[1,8,15$, $16]$, or number of blood vessels in the whole specimen $[18,21]$. To minimize the influence of SCZ thickening on microvascular density, we recommend using the mean value of the number of capillaries and PCVs per unit area at five randomly selected sites.

\section{Typical peritoneal morphology}

Upon obtaining a specimen using the appropriate biopsy strategy, there are no artificial damages such as abraded appearance and denudation of mesothelial cells in the peritoneal surface. Additionally, the structure of SCZ and deep fatty layer is maintained without any distortion or shrinkage (Fig. 4). On the contrary, upon inappropriate collection of a specimen, such as being sloppily treated with the tweezers, the abovementioned artificial findings will be observed (Fig. 5), causing difficulties in the genuine evaluation of the morphological changes in the peritoneum. Figure 6 shows an example of peritoneal morphology of a specimen appropriately obtained from patients clinically diagnosed EPS with hyalinizing peritoneal sclerosis. Specifically, the specimen shows the following characteristics: mesothelial cell denudation, remarkable SCZ thickening with collagenous tissue degeneration, arteriolar calcification, and capillary and PCV obliteration. Cellular infiltration and new membrane formation, occasionally observed in EPS

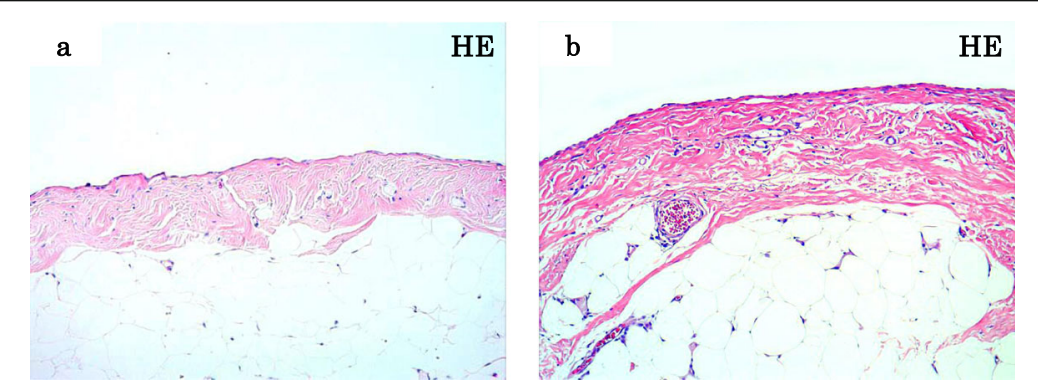

Fig. 4 Peritoneal histology sampled appropriately. a Peritoneum sampled at the initiation of peritoneal dialysis (PD) therapy. b Peritoneum sampled at PD catheter removal. These samples show no artificial damage of the peritoneal surface, distortion, or shrinkage. HE: Hematoxylin and eosin staining 


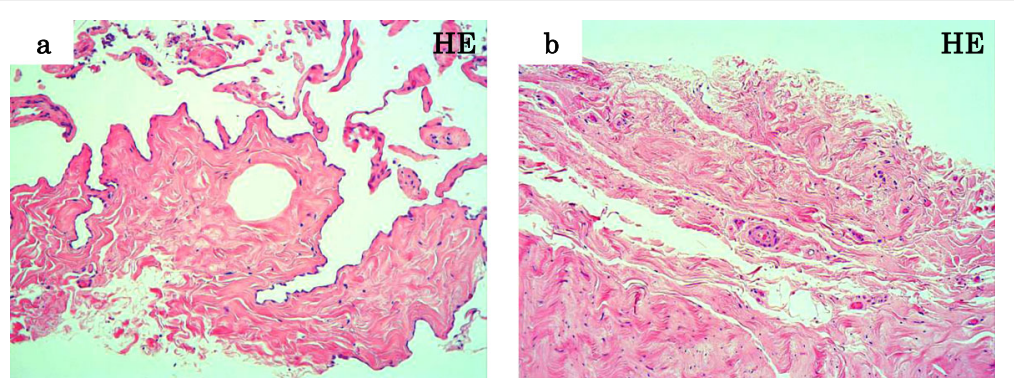

Fig. 5 Peritoneal histology presenting with difficulty in evaluation.a Peritoneum sampled at initiation of peritoneal dialysis (PD) therapy. This sample shows shrinkages, denudation of the submesothelial compact zone, and scattered mesothelial cells. b Peritoneum sampled at PD catheter removal. This sample shows an abraded appearance of peritoneal surface and denudation of mesothelial cells. HE: Hematoxylin and eosin staining

peritoneum, especially during early stages, are not observed in this specimen.

\section{Discussion}

Major problems associated with peritoneal biopsy include inadequate quantity of specimen and difficulty in differentiating artifacts from the morphological changes caused by PD. To perform an appropriate histological examination of the peritoneum, adequate care is required during peritoneal sampling to avoid mechanical damage to the peritoneum. Evaluation of the peritoneal morphology becomes difficult if the peritoneal sample comes in direct contact with the peritoneal surface or electrocautery or is excessively stretched at fixation. In a previous study, von Ruhland et al. [7] conducted a

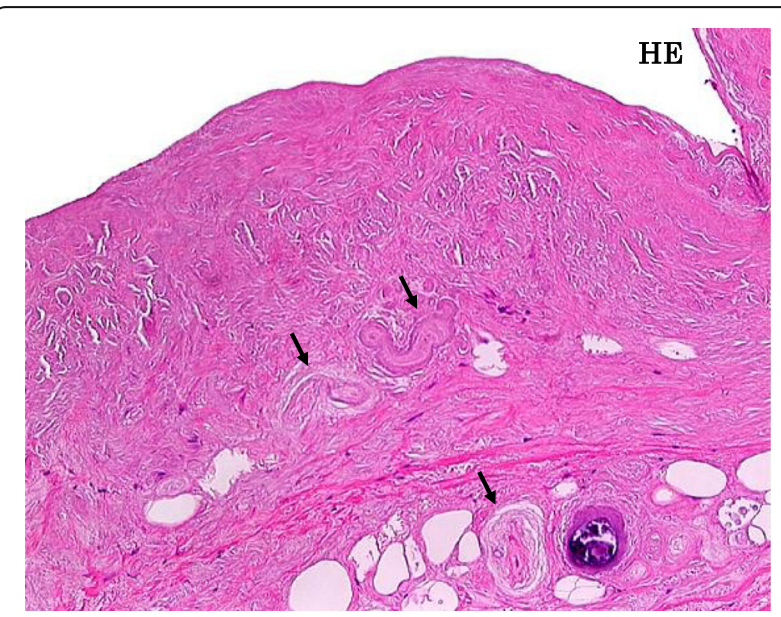

Fig. 6 Peritoneal histology sampled appropriately from a patient with clinically diagnosed encapsulating peritoneal sclerosis. This sample is characterized by the following features: mesothelial cell denudation, remarkable submesothelial compact zone thickening with collagenous tissue degeneration, arteriolar calcification, and capillary and post-capillary venule (PCV) obliteration. Cellular infiltration and new membrane formation do not appear in this sample. Arrows show capillary and PCV obliteration. HE: Hematoxylin and eosin staining simulation of various types of surgical trauma to the rat peritoneum. The authors demonstrated that peritoneal mesothelial cells could undergo degeneration simply following exposure to ambient air or massage of the peritoneum; furthermore, this change resembled the peritoneal surface sampled from a patient undergoing PD. An additional issue resulting from adding the peritoneal biopsy procedure is the potential risk for internal organ damage or prolongation of operative time. Thus, it is necessary to establish a biopsy method that enables to perform safely without artifacts.

The method proposed here can be performed easily without the need for specialized surgical instruments. Additionally, an adequate quantity of samples can be obtained under vision with little mechanical damage to the specimen. At our institution, 166 biopsies have been performed to date using this method, and complication such as internal tract damage has not been observed. Unfortunately, our outcomes cannot be compared to those at other institutions due to the lack of reports on complications related to peritoneal biopsy. Because prolongation of the operative time associated with our procedure is 10 min at most, this disadvantage is thought to be minimal.

As mentioned earlier, to date, only one study has addressed the methodology and proposal of peritoneal biopsy [7]. Similar to the method proposed by von Ruhland et al. [7], our method carries a risk of causing intraperitoneal injury at the time of exfoliation and incision of the peritoneal sample. However, we believe that such a risk is lower because peritoneal sampling is possible under vision once the abdominal cavity is reached. Furthermore, the part of the specimen $>1 \mathrm{~mm}$ from the edge toward the center need not be touched and pulled excessively when placing on the cellophane using our method. These procedures lead to a simpler operation and a more precise evaluation of the central part of the specimen compared with the previously published method. Our proposal that the duration between sampling and placing the specimen into fixative should be 
within 5 min is based on this previous study [7]. The authors reported that air drying for 5 min produced obvious changes to the appearance and density of microvilli in the mesothelium [7]. Based on these results, we set the required time for biopsy.

Additionally, there is a need to establish a biopsy area and fixation method for precise morphological examination of biopsy specimens. In an earlier study on experimental PD rat models, Duman et al. [10] reported that the peritoneum obtained from the anterior abdominal wall was the most affected area and that there were no significant differences between fixation with $4 \%$ formaldehyde and B5 solution. Referring to this article, we sampled the parietal peritoneum of the anterior abdominal wall near the surgical wound, and the sampling site becomes almost always the same. At our institution, a fixative containing $10 \%$ formalin has been used, and no difficulty in evaluation has been reported to date.

Currently, there are a few multicenter studies reporting on the difficulty of biopsy. In a multicenter study, Honda et al. [2] have evaluated morphologic changes in the peritoneum of patients with renal failure including those undergoing PD. The authors have reported that evaluation was possible in 71 of 173 specimens for SCZ thickness and 139 of 173 specimens for vasculopathy. Schaefer et al. [23] have evaluated peritoneal morphology in normal subjects and reported that 35 of 142 specimens were not available for evaluation due to inadequate sample quality; furthermore, they have reported that mesothelial cell denudation was observed in 22 of $106(21 \%)$ parietal peritoneum samples, possibly due to preservation artifacts [23]. These reports indicate that appropriate sampling of the peritoneum is important for precise morphological evaluation, especially in multicenter studies.

There are several limitations in our biopsy procedure. First, only the parietal peritoneum near the PD catheter insertion area can be obtained using this method. To obtain the parietal peritoneum from other areas or to obtain the visceral peritoneum, major surgery with a significant incision of the abdominal wall or laparoscopic surgery is required. Because such surgeries are more invasive and may lead to mechanical injury following electrocautery treatment, it is necessary to obtain informed consent and to weigh the benefits against the associated risks. Second, it is not possible to avoid extending the skin incision 1 $\mathrm{cm}$ compared with the normal PD catheter surgery. However, we believe that this has little influence on a patient's burden and wound healing. Third, informed consent for the biopsy should be carefully obtained. Specifically, it is emphasized that this visual biopsy method is safe and that histological peritoneal evaluation is essential to predict the risk for EPS. Finally, this proposal is derived from improvements incorporated into practice based on our experience. Because there are only a few reports regarding a precise biopsy procedure, it is necessary to perform a multicenter study or a questionnaire survey in the future to verify whether this method is the best.

In conclusion, histological assessments of the peritoneal membrane using peritoneal biopsy samples are fundamental for the evaluation of the peritoneal damage caused by PD, as well as for the diagnosis or prediction of EPS. Therefore, there is a need to establish a biopsy procedure that can be safely performed without artifacts and difficulties. Our proposed procedure allows for quickly and carefully obtaining the peritoneum, resulting in a minimal surgical invasiveness to the patient and a precise morphological evaluation.

\section{Abbreviations \\ EPS: Encapsulating peritoneal sclerosis; GDP: Glucose degradation product; PCV: Post-capillary venule; PD: Peritoneal dialysis; SCZ: Submesothelial compact zone}

\section{Acknowledgements}

Not applicable

\section{Authors' contributions}

HK participated in the design of this study. HK and MM helped to draft the manuscript. All authors read and approved the final manuscript.

\section{Funding}

Not applicable

Availability of data and materials

Not applicable

\section{Ethics approval and consent to participate}

This study received ethics approval in Fukuoka Red Cross Hospital (Fukuoka city, Japan), and peritoneal tissue samples were obtained from patients in the same hospital. All patients provided written consent to participate.

\section{Consent for publication}

Not applicable

\section{Competing interests}

The authors declare that they have no competing interests.

\section{Author details}

${ }^{1}$ Department of Nephrology, Kyushu Central Hospital of the Mutual Aid Association of Public School Teachers, Fukuoka, Japan. ${ }^{2}$ Department of Anatomy, Showa University School of Medicine, Tokyo, Japan. ${ }^{3}$ Department of Pathology and Microbiology, Faculty of Medicine, Saga University, Saga, Japan. ${ }^{4} J u n t e n d o$ Advanced Research Institute for Health Science, Tokyo, Japan. ${ }^{5}$ Miyazaki Clinic, Nagasaki, Japan. ${ }^{6}$ Division of Nephrology and Rheumatology, Department of Internal Medicine, Aichi Medical University School of Medicine, Nagakute, Japan. ${ }^{7}$ Division of Kidney and Hypertension Department of Internal Medicine, The Jikei University of Medicine, Tokyo, Japan. ${ }^{8}$ Department of Medicine and Clinical Science, Graduate School of Medical Sciences, Kyushu University, Fukuoka, Japan. ${ }^{9}$ Center for Advanced Integrated Renal Science, Tohoku University Graduate School of Medicine, Sendai, Japan.

Received: 18 July 2019 Accepted: 23 December 2019

Published online: 15 January 2020

\section{References}

1. Williams JD, Craig KJ, Topley N, von Ruhland C, Fallon M, Newman GR, et al. Morphologic Changes in the peritoneal membrane of patients with renal disease. J Am Soc Nephrol. 2002;13:470-9.

2. Honda K, Hamada C, Nakayama M, Miyazaki M, Sherif AM, Harada T, et al. Impact of uremia, diabetes, and peritoneal dialysis itself on the 
pathogenesis of peritoneal sclerosis: a quantitative study of peritoneal membrane morphology. Clin J Am Soc Nephrol. 2008;3:720-8.

3. Hamada C, Honda K, Kawanishi K, Nakamoto H, Ito Y, Sakurada T, et al. Morphological characteristics in peritoneum in patients with neutral peritoneal dialysis solution. J Artif Organs. 2015;18:243-50.

4. Nomoto Y, Kawaguchi Y, Kubo H, Hirano H, Sakai S, Kurokawa K. Sclerosing encapsulating peritonitis in patients undergoing continuous ambulatory peritoneal dialysis: a report of the Japanese sclerosing encapsulating peritonitis study group. Am J Kidney Dis. 1996;28:420-7.

5. Kawanishi H, Kawaguchi Y, Fukui H, Hara S, Imada A, Kubo H, et al. Encapsulating peritoneal sclerosis in Japan: a prospective, controlled, multicenter study. Am J Kidney Dis. 2004;44:729-37.

6. Nakayama M, Miyazaki M, Honda K, Kasai K, Tomo T, Nakamoto H, et al. Encapsulating peritoneal sclerosis in the era of a multi - disciplinary approach based on biocompatible solutions: The Next - PD Study. Perit Dial Int. 2014;34:766-74.

7. von Ruhland CJ, Newman GR, Topley N, Williams JD. Can artifact mimic the pathology of the peritoneal mesothelium? Perit Dial Int. 2003;23:428-33.

8. Mizumasa T, Hirakata H, Kuroki Y, Katafuchi R, Yosueda H, Mitsuiki K, et al. Diabetes influences peritoneal morphology in uremic patients at the initiation of peritoneal dialysis. Perit Dial Int. 2013;33:175-81.

9. Mizumasa T, Hirakata H. Trauma-free method of peritoneal sample collection and processing for evaluation genuine pathology. Jin To Toseki. 2014;77:76-8 (Japanese)

10. Duman $\mathrm{S}$, Sen $\mathrm{S}$. Technical aspect in studying peritoneal morphology in animal models of peritoneal dialysis. Perit Dial Int. 2009;29(Suppl 2):40-4.

11. Oka H, Yamada S, Kamimura T, Hara M, Hirashima Y, Matsueda S, et al. Modified simple peritoneal wall anchor technique (PWAT) in peritoneal dialysis. Perit Dial Int. 2017;37:103-8.

12. Plum J, Hermann S, Fussholler A, Schoenicke G, Donner A, Rohrborn A, et al. Peritoneal sclerosis in peritoneal dialysis related to dialysis settings and peritoneal transport properties. Kidney Int. 2001;59(Suppl 78):42-7.

13. Ayuzawa N, Ishibashi Y, Takazawa Y, Kume H, Fujita T. Peritoneal morphology after long-term peritoneal dialysis with biocompatible fluid: Recent clinical practice in Japan. Peit Dial Int. 2012;32:159-67.

14. Kawanishi K, Honda K, Tsukada M, Oda H, Nitta K. Neutral solution low in glucose degradation products is associated with less peritoneal fibrosis and vascular sclerosis in patients receiving peritoneal dialysis. Perit Dial Int. 2013;33:242-51.

15. Tawada M, Ito Y, Hamada C, Honda K, Mizuno M, Suzuki Y, et al. Vascular endothelial cell injury is an important factor in the development of encapsulating peritoneal sclerosis in long-term peritoneal dialysis patients. PLoS One. 2016;11:e0154644.

16. Sherif AM, Nakayama M, Maruyama Y, Yoshida H, Yamamoto H, Yokoyama $\mathrm{K}$, et al. Quantitative assessment of the peritoneal vessel density and vasculopathy in CAPD patients. Nephrol Dial Transplant. 2006;21:1675-81.

17. Sherif AM, Yoshida H, Maruyama Y, Yamamoto H, Yokoyama K, Hosoya T, et al. Comparison between the pathology of encapsulating sclerosis and simple sclerosis of the peritoneal membrane in chronic peritoneal dialysis. Ther Apher Dial. 2008;12:33-41.

18. Shimaoka T, Hamada C, Kaneko K, lo H, Sekiguchi Y, Aruga S, et al. Quantitative evaluation and assessment of peritoneal morphologic changes in peritoneal dialysis patients. Nephrol Dial Transplant. 2010;25:3379-85.

19. Honda K, Nitta K, Horita S, Yumura W, Nihei H, Nagai R, et al. Accumulation of advanced glycation end products in the peritoneal vasculature of continuous ambulatory peritoneal dialysis patients with low ultra-filtration. Nephrol Dial Transplant. 1999;14:1541-9.

20. Mateiisen MAM, van der Wal AC, Hendriks PMEM, Zweers MM, Mulder J, Struijk DG, et al. Vascular and interstitial changes in the peritoneum of CAPD patients with peritoneal sclerosis. Perit Dial Int. 1999;19:517-25.

21. Combet S, Miyata T, Moulin P, Pouthier D, Goffin E, Devuyst O. Vascular proliferation and enhanced expression of endothelial nitric oxide synthase in human peritoneum exposed to long-term peritoneal dialysis. J Am Soc Nephrol. 2000;11:717-28.

22. Numata M, Nakayama M, Nimura S, Kawakami M, Lindholm B, Kawaguchi Y. Association between an increased surface area of peritoneal microvessels and a high peritoneal solute transport rate. Perit Dial Int. 2003;23:116-22.

23. Schaefer B, Bartosova M, Macher-Goeppinger S, Ujszaszi A, Wallwiener M, Nyarangi-Dix J, et al. Quantitative histomorphometry of the healthy peritoneum. Sci Rep. 2016. https://doi.org/10.1038/srep21344.

\section{Publisher's Note}

Springer Nature remains neutral with regard to jurisdictional claims in published maps and institutional affiliations.

\section{Ready to submit your research? Choose BMC and benefit from:}

- fast, convenient online submission

- thorough peer review by experienced researchers in your field

- rapid publication on acceptance

- support for research data, including large and complex data types

- gold Open Access which fosters wider collaboration and increased citations

- maximum visibility for your research: over $100 \mathrm{M}$ website views per year

At BMC, research is always in progress.

Learn more biomedcentral.com/submissions 